\title{
Software Technologies in Satellite Monitoring of the Arctic Ice Cover
}

\author{
Vasiliy V. Asmus*a, \\ Vladimir A. Krovotyntsev and Valeriy P. Pyatkin ${ }^{\mathrm{b}}$ \\ ${ }^{a}$ State Research Center of Space Hydrometeorology «Planeta», Rosgidromet \\ 7 Bolshoi Predtechenskii Str., Moscow, 123242, Russia \\ ${ }^{b}$ Institute of Computational Mathematics \\ and Mathematical Geophysics SB RAS \\ 6 Akademika Lavrenteva, Novosibirsk, 630090, Russia
}

Received 05.02.2015, received in revised form 30.03.2015, accepted 29.06.2015

These measurements of polar-orbiting Russian and foreign satellite remote sensing (RS) were used to monitor ice cover in the Arctic. To solve this problem FGBU «SIC» Planet «and ICM and $M G S B R A S$ are carrying out long-term cooperation in the development and use of methods and technologies for satellite data processing. The report provides data on the current and future domestic and foreign satellites, including space system "Arktika», the description of the developed methods and operational monitoring technologies ice, examples of satellite information products on the territory of the Arctic.

Keywords: remote sensing, satellite data,ice cover monitoring, software technologies.

DOI: 10.17516/1999-494X-2015-8-6-680-689.

\section{Программные технологии в космическом мониторинге ледяного покрова Арктики}

\author{
В.В. Асмус ${ }^{a}$, В.А. Кровотынцев ${ }^{a}$, В.П. Пяткин ${ }^{\sigma}$ \\ ${ }^{a}$ НИЦ космической гидрометеорологии «Планета» \\ Россия, 123242, Москва, Большой Предтеченский пер., 7 \\ ${ }^{6}$ Институт вычислительной математики \\ и математической геофизики СО РАН \\ Россия, 630090, Новосибирск, пр. Лаврентьева, 6
}

Данные измерений полярно-орбитальных российских $u$ зарубежных спутников дистанционного зондирования Земли (ДЗ3) были использованы для мониторинга ледяного

(c) Siberian Federal University. All rights reserved

* Corresponding author E-mail address: asmus@planet.iitp.ru 
покрова Арктики. В рамках решения этой задачи ФГБУ «НИЦ «Планета» и ИВМ и МГ СО РАН осуществляют долговременное сотрудничество в области создания и использования методов и технологий обработки спутниковых данных. В докладе приводятся данные о действующих и перспективных отечественных и зарубежных космических аппаратах, в том числе космической системы "Арктика», описание разработанных методов и оперативных технологий мониторинга ледяного покрова, примеры спутниковой информационной продукции по территории Арктики.

Ключевые слова: дистанционное зондирование, спутниковые данные, мониторинг ледяного покрова, программные технологии.

\section{1. Введение}

Наблюдение за состоянием ледяного покрова Земли является одной из традиционных задач гидрометеорологии, климатологии и в целом мониторинга состояния окружающей среды. Оперативная информация о пространственном распределении, дрейфе, типе, возрасте, концентрации морского льда и айсбергов необходима для обеспечения безопасности навигации, рыболовства, добычи нефти и газа в полярных районах, а также для составления ледовых прогнозов различной заблаговременности. С точки зрения климатологии чрезвычайно важно накопление и анализ многолетних рядов данных о различных характеристиках ледяного покрова, являющихся индикаторами изменений регионального и глобального климата. В течение длительного времени ФГБУ «НИЦ «Планета» и ИВМиМГ СО РАН совместными усилиями разрабатывают представительный набор программных технологий, позволяющих решать различные задачи обработки данных дистанционного зондирования Земли (ДЗ3) [1-3]. В соответствующем программном комплексе реализованы программные технологии обработки многоспектральной спутниковой информации оптического, инфракрасного и микроволнового диапазонов, ориентированные на мониторинг ледяного покрова Арктики.

\section{2. Космические системы мониторинга ледяного покрова Земли}

Космический мониторинг ледяного покрова Арктики проводится в ФГБУ «НИЦ «Планета» с момента запуска в 1983 г. первого отечественного океанографического спутника серии «Океан» с радиолокационной станцией бокового обзора Х-диапазона (длина волны 3,2 см). Спутниковые радиолокационные данные являются наиболее эффективным средством наблюдения ледяного покрова, так как результаты космической съемки не зависят от освещенности и облачного покрова. Наряду с радиолокационными данными для осуществления ледовых наблюдений в разные годы брали также данные видимого и инфракрасного диапазонов с отечественных и зарубежных космических аппаратов (КА) серии «Метеор», «Ресурс», NOAA, MetOp, EOS (TERRA, AQUA) и др. В настоящее время для мониторинга ледяного покрова активно используют данные с российского метеорологического спутника «Метеор-М» № 1 (запущен 17.09.2009 г.). Группировка спутников серии «Метеор-М» должна пополниться еще двумя космическими аппаратами (один из которых океанографический), и с этого времени на орбитах постоянно должны функционировать два КА метеорологического и один КА океанографического назначения. Создаётся также группировка в составе двух метеорологических спутников на геостационарных орбитах серии «Электро», первый из спутников этой группировки «Электро-Л» был запущен 20 января 2011 г. Кроме того, формируется группировка из

$$
-681-
$$


двух космических аппаратов для контроля чрезвычайных ситуаций серии «Канопус $-\mathrm{B} », \mathrm{a}$ также из двух природно-ресурсных спутников серии «Ресурс - П». В дополнение осуществляется также разработка уникальной (не имеющей мировых аналогов) системы спутников на высокоэллиптических орбитах - МКС «Арктика», предназначенной для мониторинга северного полярного региона. Арктический регион физически недоступен для наблюдений спутниками международной базовой метеорологической группировки на геостационарных орбитах. Зона качественного мониторинга с геостационарных орбит ограничивается зенитным углом наблюдения 70 градусов, что соответствует широте 60 градусов. Каналы связи, размещенные на геостационарных спутниках, не могут обеспечить качественный прием данных с арктических дрейфующих буёв и автоматических гидрометеостанций. Контроль состояния ледяного покрова Арктики осуществляли на основе данных, принимаемых наземным комплексом приема и обработки спутниковой информации, размещенном в ФГБУ «НИЦ «Планета», включающим в себя три центра: Европейский (Москва, Обнинск, Долгопрудный); Сибирский (Новосибирск) и Дальневосточный (Хабаровск), что обеспечивало полное покрытие космической съемкой всей территории Северного морского пути и всех замерзающих морей России. Еще одним источником информации, используемой для обеспечения мониторинга ледяного покрова Арктики, является получение оперативных спутниковых данных по международной системе EARS (EUMETSAT Advanced Retransmission Service). В 2009 г. (в соответствии с соглашением Росгидромет - EUMETSAT) ФГБУ «НИЦ «Планета» вошел в состав международной системы EARS. Для этой цели в ФГБУ «НИЦ «Планета» первоначально был создан и введен в действие комплекс обработки и оперативного обмена информацией с крупными мировыми спутниковыми центрами. Это позволило обеспечить оперативный доступ через систему EumetCast к данным спутникового зондирования по Северному полушарию Земли, принимаемым в крупных спутниковых центрах Европы и мира.

\section{3. Предварительная обработка спутниковых данных}

На протяжении многих лет в ФГБУ «НИЦ «Планета» и в ИВМиМГ СО РАН создавались, развивались и совершенствовались методы и технологии обработки спутниковых данных. В частности, были разработаны методы относительной и абсолютной калибровки данных РЛС БО ИСЗ серии «Океан», позволяющие осуществить переход от значений яркости радиолокационных изображений к физической характеристике ледяного покрова - удельной эффективной площади рассеяния (УЭПР). С применением данных методов была проведена оценка пространственно-временных изменений и угловых зависимостей значений УЭПР многолетнего и однолетнего льда в двух (отличающихся по своим климатическим особенностям) районах Арктики [3]. Результаты исследований характеристик радиолокационного обратного рассеяния морского льда использовались при создании технологии бесшовного соединения спутниковых радиолокационных изображений при построении обзорных монтажей (мозаик) Арктики или ее отдельных территорий. Обзорные радиолокационные мозаики могут быть применены на практике как самостоятельная информационная продукция либо служить в качестве исходных данных для построения карт ледовой обстановки. Следующим важным направлением в обеспечении космического мониторинга является создание технологии построения карт ледовой обстановки по спутниковым данным. В технологии воплощено 
сочетание автоматизированных и интерактивных процессов. В автоматизированном режиме осуществляется предварительная обработка спутниковых изображений, которая включает в себя набор общепринятых операций по яркостным и контрастным преобразованиям изображений. Программный комплекс включает ряд основанных на видоизменении гистограмм контрастных преобразований. В частности, реализован механизм референтного выравнивания [1], при котором яркостные и контрастные характеристики изображений приводятся либо к характеристикам некоторого изображения, выбранного в качестве референтного (базового), либо к характеристикам распределения Гаусса с заданными параметрами. Этот этап необходим в тех случаях, когда интересующий исследователя участок земной территории покрывается несколькими разновременными космическими снимками, полученными с разными условиями съемки. В группу геометрических преобразований включено масштабирование изображений и отображение космических снимков на растровые карты (геокодирование). Трансформирование спутниковых изображений в картографическую основу является геометрическим преобразованием, отображающим весь снимок либо его часть на заранее подготовленную растровую географическую карту соответствующей территории. Карта строится в выбранной географической проекции с использованием различных баз данных (в том числе шейп-файлов ГИС ArcView) для нанесения на лист карты контурных элементов пространственных объектов. Отображение снимка на карту основано на использовании идентичных опорных точек снимка и карты. Возможно использование одного из двух типов отображений: на основе применения семейства кусочно-аффинных преобразований, которые строятся на множестве треугольников снимка и карты, получаемых в результате триангуляции выпуклой оболочки множества опорных точек снимка, и на основе отображающего полинома заданной степени (первой, второй или третьей). Мозаики спутниковых изображений формируются путем объединения трансформированных на единую картографическую основу изображений. В интерактивном режиме осуществляется дешифрирование на космических снимках ледовых параметров (возраст, сплоченность, формы льда, обобщенные характеристики и др.) и их представление на карте в соответствии с требованиями ВМО. На рис. 1 представлена карта-схема ледовой обстановки в Арктике.

\section{4. Технология распознавания}

Центральные вопросы тематической обработки (интерпретации) данных ДЗ3 - вопросы повышения качества дешифрирования - непосредственно связаны с проблемой выбора адекватных алгоритмов распознавания [2]. Современный опыт автоматизированного распознавания данных ДЗ3 показывает, что заранее практически невозможно установить, какой алгоритм будет лучше с точки зрения точности классификации. Поэтому в распознающую систему целесообразно закладывать несколько алгоритмов и выбор оптимального алгоритма проводить эмпирически. В классификации данных ДЗ3 чаще других используют методы, которые можно разбить на две группы: контролируемая классификация (классификация с обучением) и кластеризация (классификация без обучения). В классификации с обучением для распределения векторов признаков по представляющим интерес классам применяем процесс обучения классификатора способности различать эти классы на основе репрезентативных выборок представителей классов. Эти выборки называются обучающими. Процесс обучения фактически является

$$
-683-
$$



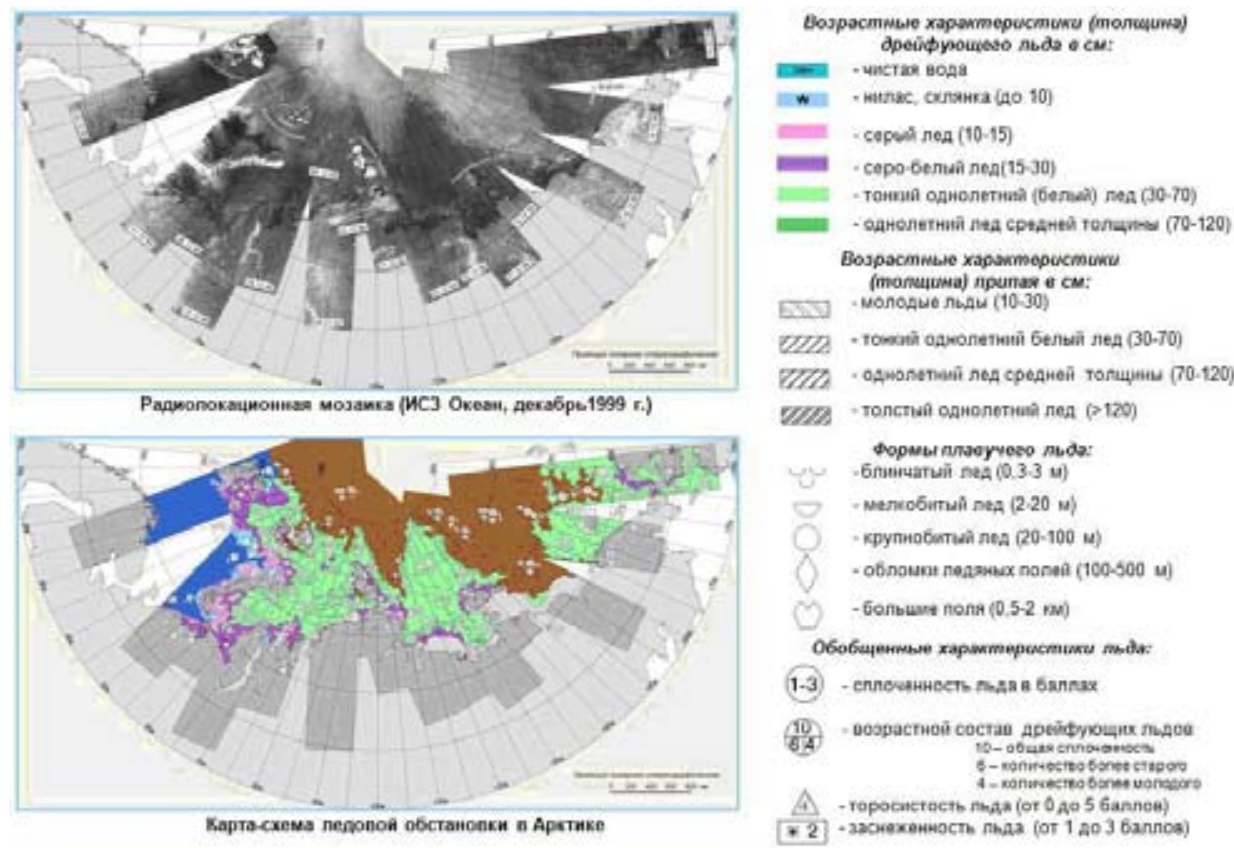

Рис. 1

процессом построения функций правдоподобия (или решающих функций) для классов. Эти функции будут затем использоваться для классификации каждого вектора признаков, принадлежащих только одному (жесткая классификация) либо нескольким классам (нечеткая классификация). Обучение классификатора может быть проконтролировано путем определения вероятности правильной классификации (для этого часть из обучающих выборок переводится в разряд контрольных). Контролируемая классификация в программном комплексе Planeta Monitoring основана на использовании байесовской стратегии максимального правдоподобия для нормально распределенных векторов признаков. Пусть $x-N$-мерный вектор признаков $x=\left(x_{1}, \ldots x_{N}\right)^{T}$, где $N$ - число спектральных диапазонов. Предполагается, что векторы $x$ имеют в классе $\omega_{i}$ нормальное распределение $N\left(m_{i}, B_{i}\right)$ со средним $m_{i}$ и ковариационной матрицей $B_{i}$. В этом случае байесовская стратегия максимального правдоподобия для поэлементного классификатора формулируется следующим образом [2].

Пусть $\Omega=\left(\omega_{1}, \ldots, \omega_{m}\right)$ - конечное множество классов, $p\left(\omega_{i}\right)$ - априорная вероятность класса $\omega_{i}$. Тогда решающая функция класса $\omega_{i}$ имеет вид

$$
g_{i}(x)=\ln \left(p\left(\omega_{i}\right)\right)-0.5 \ln \left(\left|B_{i}\right|\right)-0.5\left(x-m_{i}\right)^{T} B_{i}^{-1}\left(x-m_{i}\right) .
$$

Классическое решающее правило для жесткой классификации принимает следующий вид: вектор $x$ заносится в класс $\omega_{i}$, если $g_{i}(x)>g_{j}(x)$ для всех $j \neq i$.

Поскольку физические размеры реально сканируемых пространственных объектов, как правило, больше разрешения съемочных систем, между векторами признаков существуют взаимосвязи [2]. Использование информации подобного рода дает возможность повысить точность классификации, если пытаться распознавать одновременно блок смежных векторов ква- 
дратной или крестообразной формы. Будем называть такой блок векторов объектом. Рассмотрим объект $X=\left(x_{1}, \ldots x_{L}\right)^{T}$, состоящий из смежных $N$-мерных векторов $x_{i}, i=1, \ldots, L$ (например, в окрестности $3 * 3,5 * 5, \ldots$ элементов). Решение об отнесении центрального элемента объекта к тому или иному классу принимается на основе результата классификации всего объекта. Такой подход порождает целое семейство решающих правил. Во-первых, это использование принципа голосования, т.е. независимая классификация элементов объекта и отнесение центрального элемента к тому классу, которому было отнесено большинство элементов объекта. Во-вторых, это применение текстурных операторов (простейший пример - описание объекта $X$ через вектор средних составляющих его элементов) с последующим отнесением центрального элемента к классу, к которому был отнесен параметр, характеризующий $X$. В-третьих, описание объекта $X$ случайным марковским полем.

В состав программного комплекса входит реализация классического алгоритма жесткой кластеризации - алгоритма $K$-средних, широко используемого для разбиения на кластеры больших объемов многомерных данных [2]. Алгоритм $K$-средних может быть отнесен к классу параметрических, так как он неявным образом предполагает природу плотности вероятности: кластеры стремятся иметь конкретную геометрическую форму, зависящую от выбранной метрики. Используются следующие метрики: Евклидова, Махаланобиса, Чебышева, city-block-расстояние. Известно также, что результат кластеризации методом $K$-средних зависит от задания начальных центров кластеров. Предоставляется выбор одного из трех вариантов, два из которых определяются на основе статистических характеристик набора данных и один основан на случайной выборке. Другой подход, позволяющий получать разбиение векторов измерений на кластеры произвольной формы, основан на предположении, что исходные данные являются выборкой из многомодового закона распределения, причем векторы, отвечающие отдельной моде, образуют кластер. Таким образом, задача сводится к анализу мод многомерных гистограмм. На рис. 2 представлены этапы технологии автоматизированного распознавания ледяных объектов по многозональным спутниковым данным.

\section{5. Построение полей дрейфа морского льда}

Еще одним примером сотрудничества ФГБУ «НИЦ «Планета» с ИВМиМГ СО РАН является экспериментальная технология построения полей дрейфа морского льда. В технологии использован метод восстановления полей дрейфа морского льда по опознанным изменениям положения некоторых ледовых объектов (трассеров) на разновременных и картографически совмещенных спутниковых изображениях. Определение координат перемещения ледяных полей с заданным шагом достигалось в результате использования аппроксимаций по методу триангуляции Делоне. При этом на спутниковых изображениях предварительно производилось закрепление контуров береговой черты с помощью опорных точек для того, чтобы на картах дрейфа льда произвести разделение неподвижной суши и перемещающегося плавучего льда. Одним из способов определения пространственных перемещений ледяных полей по разновременным спутниковым изображениям служит метод, основанный на нахождении максимумов коэффициента взаимной корреляции. Реализован соответствующий алгоритм и имеются результаты вычислительных экспериментов на изображениях, полученных с KA «METEOSAT-8». На рис. 3

$$
-685-
$$




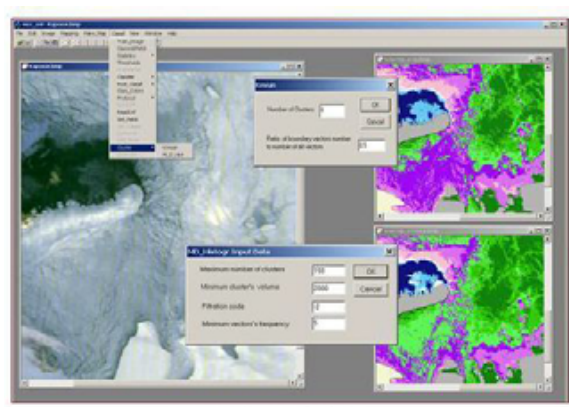

Кластерный анализ

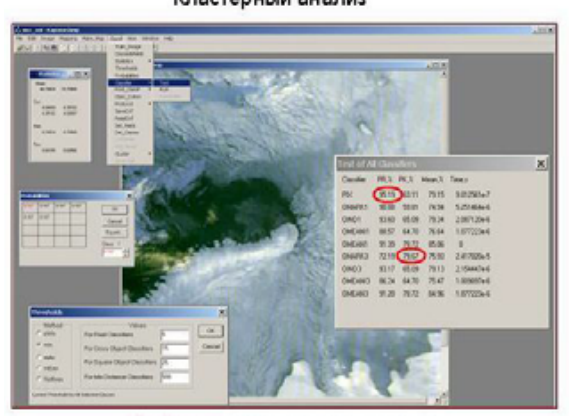

Выбор метода распознавания

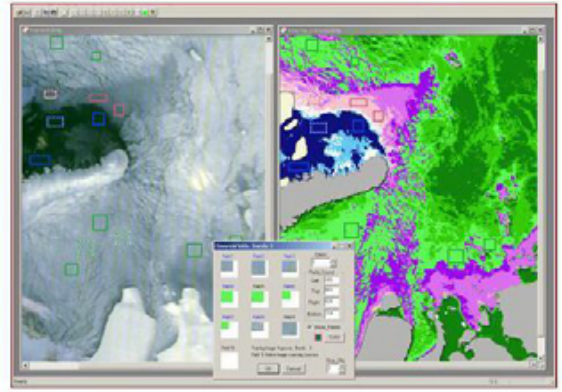

Выбор тестовых участков

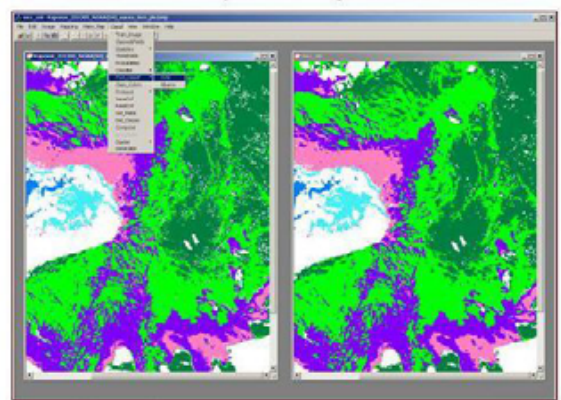

Классификация и сглаживание результата

Рис. 2

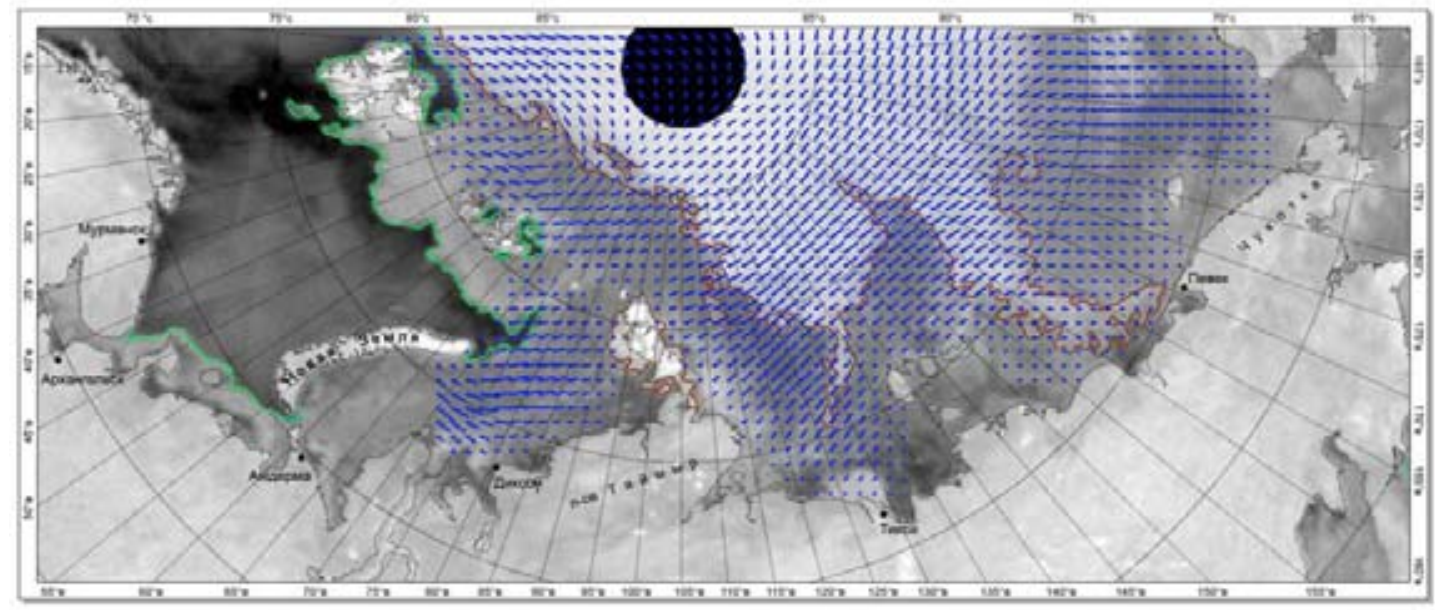

Рис. 3

представлено построение карты дрейфа многолетнего льда в российском секторе Арктики (по данным ИС3 QuikScat, SeaWind NRT, разрешение 35-40 км, 14-25 февраля 2005 г.).

\section{6. Методы линеаментного анализа}

При обработке космических снимков для обнаружения на них объектов, представляющих интерес (в частности, линеаментов), в силу целого ряда причин предпочтение отдается статистическому подходу [4]. Основная причина состоит в том, что вследствие случайного 

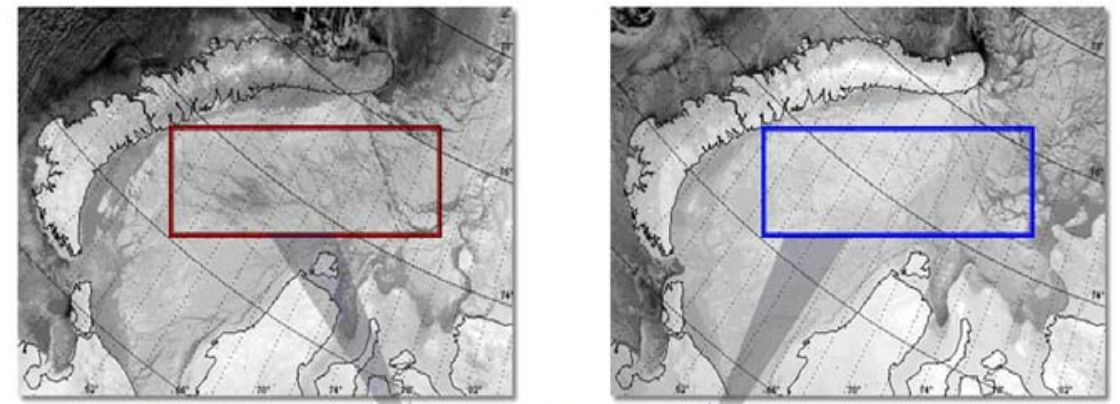

Выделение линеаментов размером 30-80 км на фрагментах изображений

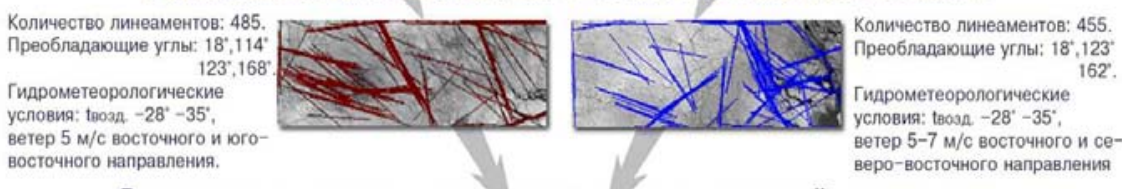

Распределение линеаментов как индикатор пространственной структуры льда

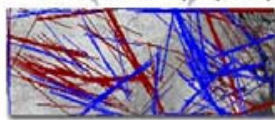

В восточной части исследуемой территории увеличилась зона разряжения ледяного покрова, в западной части произошло сжатие и смещение ледяных полей в южном и юго-западном направлении со скоростью $0,7 \mathrm{~km} / \mathrm{cy}$.

Рис. 4

характера природных процессов данные дистанционных измерений (спектросовмещенные изображения) содержат много случайных вариаций, маскирующих различия значений яркости изображения в точках области объекта и в точках области фона. Совместными усилиями ФГБУ «НИЦ «Планета» и ИВМиМГ СО РАН произведена адаптация методов линеаментного анализа (ранее использовавшегося для изучения структур суши в интересах геологических исследований [4]) для изучения структуры морского льда в Арктике. Было установлено, что по распределению линеаментов, выделенных на разновременных спутниковых изображениях, можно оценивать упорядоченность и перемещение трещиноватых структур плавучего морского льда, а зоны повышенной плотности линеаментов, выделенные на космических снимках морского льда, в большей степени подвержены деформации. Именно в этих зонах происходит наибольшее количество сжатий и разряжений ледяного покрова. Рис. 4 представлен структурный (линеаментный) анализ фрагментов изображения ледяного покрова с использованием данных спутника NOAA, сканер AVHRR, канал: 10,5 11,5 мкм.

\section{7. Изучение многолетних изменений ледяного покрова Арктики}

Созданные технологии обработки спутниковых данных используются в ФГБУ «НИЦ «Планета» не только в оперативной практике, но и для изучения долговременных изменений характеристик ледяного покрова по архивным данным. Так, например, в 2002-2013 гг. была проведена оценка сезонных изменений границ распространения и площадей покрытия многолетнего льда в Арктике по данным скаттерометра $\mathrm{K}_{\mathrm{U}}$-диапазона (длина волны 2,2 см) SeaWinds ИС3 QuikSCAT, с ноября 2009 г. - по данным скаттерометра С-диапазона (длина волны 5,66 см)

$$
-687-
$$


ASCAT ИС3 MetOp. Именно эти характеристики многолетнего льда наиболее чувствительны к изменениям климата и могут служить индикаторами региональных и глобальных климатических изменений. Оценка сезонных изменений площади многолетнего льда проводилась в российском секторе Арктики, ограниченном $10^{\circ}$ в.д. и $160^{\circ}$ 3.д. В результате анализа сезонных изменений за период 2002-2013 гг. были выявлены некоторые закономерности. Наибольшая площадь многолетнего льда в российской Арктике приходится на сентябрь месяц. В последующие месяцы площадь многолетнего льда неравномерно уменьшается. С сентября по декабрь ежемесячная скорость изменения площади многолетнего льда в российской Арктике составляет 20-70 тыс. км²/мес, с января по май $\sim 110-140$ тыс. км²/мес. В отдельные месяцы наблюдается небольшой прирост площади многолетнего льда (как, например, в декабре 2002 г.) за счет его поступления из канадского сектора Арктики. Обращает на себя внимание существенное сокращение протяженности многолетнего льда в российском секторе Арктики в зимний период 2007-2008 гг. В июне-августе оценки изменений площадей многолетнего льда не проводили из-за плохой разделимости на радиолокационных изображениях многолетнего и однолетнего льда в это время года.

Продолжены работы по оценке межгодовых изменений площади многолетнего льда в западном секторе Арктики. Ранее такие оценки проводили на основе многолетних рядов радиолокационных данных ИСЗ серии «Океан» за период 1983-1999 гг. В 2002-2013 гг. эти ряды были дополнены данными микроволновых скаттерометров ИСЗ QuikSCAT и MetOp. Выделение границы многолетнего льда осуществлялось с использованием технологий автоматизированного распознавания и интерактивного дешифрирования. Валидация карт-схем границ распространения многолетнего льда, полученных по данным ИСЗ серии «Океан», проводилась с использованием данных авиационных наблюдений. Среднеквадратическая погрешность определения границ распространения многолетнего льда составила 8-12 км. Оценка межгодовых изменений площади многолетнего льда проводилась в западном секторе Арктики, ограниченном $40^{\circ}$ в.д. и $105^{\circ}$ в.д. Установлено, что в исследуемом районе имеют место значительные межгодовые вариации площадей от 180 до 540 тыс. км². Наибольшая площадь многолетнего льда была отмечена за этот период в декабре 1988 и 2003 гг., наименьшая - в 1985, 1999, 2005, 2006 и 2007 гг. За период наблюдения с 1983 по 2013 г. в западном секторе Арктики с учетом пропусков информации отмечается небольшой отрицательный тренд (уменьшение площади многолетнего льда $\sim$ на 5 \%). Анализ спутниковой радиолокационной информации за 2002-2013 гг. позволил построить сезонные и межгодовые вариации площадей покрытия морского льда Арктики (рис. 5).

\section{8. Заключение}

Долговременные изменения многолетнего льда в Арктике, полученные на основе спутниковых данных, выставлены на сайтах международного (под эгидой ВМО) Северо-Евразийского климатического центра: http://seakc.meteoinfo.ru и http://neacc.meteoinfo.ru и могут служить индикаторами изменения климата. Все виды информационной продукции, получаемые при проведении космического мониторинга Арктики, по мере их поступления заносятся в специализированный цифровой архив. К настоящему времени в архиве накоплены ряды спутниковых данных по различным районам Арктики за более чем 30-летний период. 

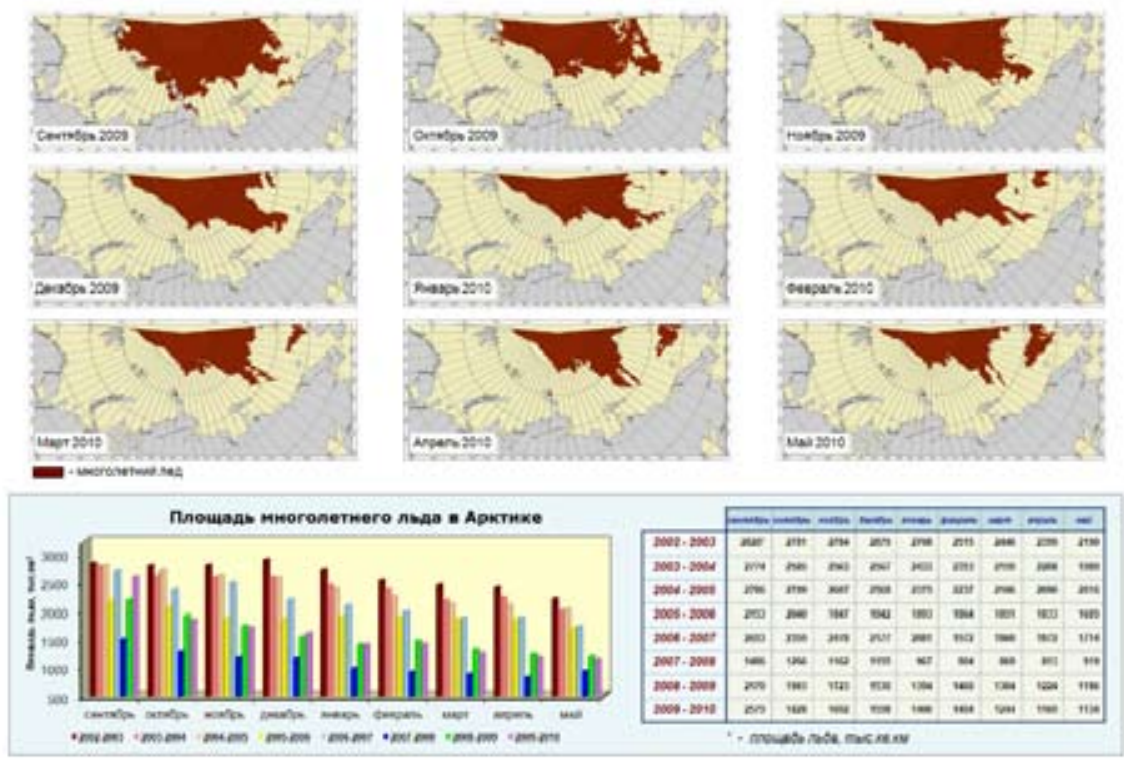

Рис. 5

Работа выполнена частично при финансовой поддержке Российского фонда фундаментальных исследований (проект № 13-07-00068) и Программы № 43 Президиума РАН (проект № 32).

\section{Список литературы}

[1] Шовенгердт Р.А. Дистанционное зондирование. Модели и методы обработки изображений. М.: Техносфера, 2010. 560 с.

[2] Асмус В.В. Автореф. дис. ... д-ра физ.-мат. наук. М., 2002. 35 с.

[3] Кровотынцев В.А., Милехин О.Е. // Исследование Земли из космоса. 1998. № 2. C. $68-80$.

[4] Пяткин В.П., Салов Г.И. // Наукоемкие технологии. 2002. Т. 3. № 3. С. 52-58. 